\title{
Outcomes Of Proximal Femur Metastases After Surgical Fixation: A Two Centres Study
}

\author{
Nur Lidawati Apandi ${ }^{1}$, Goh Kian Liang ${ }^{1}$, Mohd Shukrimi Awang ${ }^{1}$ \\ ${ }^{1}$ Department of Orthopaedics, Traumatology and Rehabilitation, Kulliyyah of \\ Medicine, International Islamic University Malaysia
}

Presenter: Nur Lidawati Apandi

Introduction: Surgery to skeletal metastases of proximal femur is important to reduce morbidity and improve quality of life. However, choice of surgery still remain debatable. This study evaluated functional outcome and survival rate in patients after skeletal reconstructive surgery. Material and Methods: Thirty patients for skeletal metastasis over the proximal femur fracture were performed in two centres. The choice of implant, complications, survival rate (using Kaplan Meier) and functional outcome (MSTS score) were evaluated. Results: Endoprosthesis was the most common procedure performed (17 patients), followed by osteosyntheses (13 patients). Total hip replacement ( 9 patients) and hemiarthroplasty (8 patients) was almost of the same proportion used as endoprosthesis implant. Plating osteosynthesis by using Dynamic Hip Screw was the most common surgical procedure (6 patients) performed followed by Interlocking Nail (4 patients) and Proximal Femoral Nail (3 patients). There were total of ten complications, of which four were due to systemic issues and six due to implant related problems. Survival rate at one year after surgery was $83.3 \%$ and at 3 years was $61.9 \%$. The mean MSTS score six months post-operative was $71 \%$. Conclusion: Patients with skeletal metastasis had a prolonged survival rate and should undergo skeletal reconstruction to reduce morbidity and improve quality of life. The surgical construct should be stable and outlast the patient to avoid further surgery. 